\title{
Building Confidential and Efficient Query Services in the Cloud with RASP Data Perturbation
}

\author{
S. Krishna Kishore ${ }^{1 *}$, Gudipati Murali ${ }^{2}$, A. Chandra Mouli ${ }^{3}$ \\ ${ }^{I}$ Research Scholar, Department of Computer Science \& Engineering, Acharya Nagarjuna University, Guntur, Andhra Pradesh. \\ Asst. Professor, Potti Sriramulu Chalavadi Mallikarjuna Rao College of Engineering \& Technology, Vijayawada, Andhra Pradesh, \\ India. \\ ${ }^{2}$ Research Supervisor, Department of Computer Science \& Engineering, Acharya Nagarjuna University, Guntur, Andhra Pradesh. \\ Professor, KKR \& KSR Institute of Technology And Sciences, Vinjanampadu, Guntur, Andhra Pradesh, India. \\ E-mail:m_gudipati@yahoo.com \\ ${ }^{3}$ Asst. Professor, Potti Sriramulu Chalavadi Mallikarjuna Rao College of Engineering \& Technology, Vijayawada, Andhra Pradesh, \\ India.E-mail:achandramouli@pscmr.ac.in \\ *Corresponding author E-mail:krishnakishoresajja@gmail.com
}

\begin{abstract}
With the improvement of administrations figuring and distributed computing, it has turned out to be conceivable to outsource extensive databases to database specialist co-ops and let the suppliers keep up the range-inquiry benefit. Nonetheless, a few information may be touchy that the information proprietor does not have any desire to move to the cloud unless the information classification and inquiry security are ensured. We propose the Random Space Encryption (RASP) approach that permits productive range look with more grounded assault versatility than existing proficiency centered methodologies. The arbitrary space irritation (RASP) information annoyance technique to give secure and proficient range question and kNN inquiry administrations for ensured information in the cloud. The RASP information annoyance strategy consolidates arrange protecting encryption, dimensionality development, arbitrary commotion infusion, and irregular projection, to give solid flexibility to assaults on the irritated information and questions. It likewise saves multidimensional reaches, which enables existing ordering systems to be connected to speedup extend question handling. The kNN-R calculation is intended to work with the RASP go inquiry calculation to process the kNNinquiries.
\end{abstract}

Keywords: Inquiry benefits in the cloud, security, run question, kNN question.

\section{Introduction}

With the wide arrangement of open distributed computing foundations, utilizing mists to have information question administrations has turned into an engaging answer for the points of interest on adaptability and cost-sparing. With the cloud frameworks, the administration proprietors can advantageously scale up or down the administration and pay for the hours of utilizing the servers. While new methodologies are expected to safeguard information secrecy and inquiry protection, the effectiveness of question administrations and the advantages of utilizing the mists ought to likewise be saved. It won't be important to give moderate inquiry benefits because of security and protection confirmation. It is additionally not useful for the information proprietor to utilize a lot of in-house assets, in light of the fact that the motivation behind utilizing cloud assets is to diminish the need of keeping up adaptable in- house frameworks. Accordingly, there is an unpredictable relationship among the information secrecy, question security, the nature of administration, and the financial aspects of utilizing the cloud.[1] Here we abridge these prerequisites for building a handy inquiry benefit in the cloud as the CPEL criteria: information classification, question security, effective inquiry handling, and low in-house preparing cost. Fulfilling these prerequisites will drastically build the multifaceted nature of developing question benefits in the cloud. Some related methodologies have been produced to address a few parts of the issue. Notwithstanding, they don't palatably address these perspectives. For instance, the crypto index and request protecting encryption (OPE) are helpless against the assaults. The upgraded crypto index approach puts substantial weight on the in-house framework to enhance the security and protection. The New Casper approach utilizes shrouding boxes to ensure information questions and inquiries, which influences the proficiency of inquiry handling and the in house workload. We propose the irregular space bother (RASP) way to deal with building viable range inquiry and $\mathrm{k}$ - closest neighbor $(\mathrm{kNN})$ question benefits in the cloud. The proposed approach will address all the four parts of the CPEL criteria and intend to accomplish a decent adjust on them. The RASP kNN question benefit (kNN-R) utilizes the RASP run inquiry administration to process kNN queries.[1] The RASP bother is an extraordinary blend of OPE, dimensionality development, arbitrary commotion infusion, and irregular projection, which gives solid privacy ensure. We have painstakingly assessed our approach with manufactured and genuine informational indexes. The outcomes demonstrate its remarkable favorable circumstances on all parts of the CPEL criteria. The RASP technique and its blend give secrecy of information and this approach is for the most part utilized to protect the multidimensional scope of inquiries in secure way, with ordering and effective question 
preparing. The go question is utilized as a part of database for recovering the put away data's. It will recover the records from the database where it can indicates some an incentive amongst upper and lower limit. The kNN question means k-Nearest Neighbor inquiry. $\mathrm{K}$ means positive number and this question are utilized to discover the estimation of closest neighbor to $\mathrm{k}$. The RASP bother installs the multidimensional information into a mystery higher dimensional space, enhanced with irregular commotion expansion to ensure the secrecy of data.[2]

\section{Related Work}

We survey the some most related techniques like OPE, crypto-file, DRE, and PIR. Request Preserving Encryption: The request protecting encryption (OPE) jam the dimensional esteem arrange after encryption. Along these lines, it can be utilized as a part of most database tasks, for example, ordering and range inquiry. OPE speaks to Order Saving Encryption is utilized for information that permits any correlation. What's more, that correlation will be connected for the scrambled information; this will be managed without unscrambling. It permits database lists to be worked over an encryption table. The disadvantage of this procedure is the encryption key is as well expansive and execution makes the time and space overhead.

Cryptoindex: Cryptoindex is additionally in light of section astute bucketization. It allots an arbitrary ID to each can; the values in the can are supplanted with the pail ID to create the helper information for ordering. To use the record for inquiry handling, a typical range question condition needs to be changed to a setconstruct inquiry in light of the pail IDs. Crypto record strategy is helpless against assaults yet the working arrangement of the crypto record has numerous troublesome procedures to give the secured encryption and security and furthermore the New Casper approach is utilized to ensure information and question yet the effectiveness of the question procedure will be influence. For instance, $\mathrm{Xi}<$ ai may be supplanted with

\section{$\mathrm{X}^{\prime} \mathrm{i} \in[$ [D1, ID2, ID3]}

On the off chance that the aggressor figures out how to know the mapping between the info unique inquiry and the yield can based question, the range that a basin ID speaks to could be evaluated. The width of the basin decides how exact the estimation should be possible. A basin dispersion plot was proposed to address this issue, which, be that as it may, needs to forfeit the exactness of question comes about. Another disadvantage of this technique is that the customer, not the server, needs to sift through the question result.

Low accuracy comes about raise extensive weight on the organize and the customer framework. Moreover, due to the randomized container IDs, the record based on basin IDs isn't so proficient for handling range questions as the file on OPE scrambled information is Separation recoverable encryption : DRE is the most instinctive strategy for protecting the closest neighbor relationship. As a result of the precisely safeguarded separations, numerous assaults can be connected. Here, speck items are utilized rather than separations to discover $\mathrm{kNN}$, which is stronger to separate focused on assaults. One disadvantage is the hunt calculation is constrained to direct sweep and no ordering strategy can be connected. Private data retrieval(PIR): PIR tries to completely protect the security of access design, while the information may not be encoded. PIR plans are ordinarily expensive.

This protection safeguarding multi catchphrase seek depends on the plain content pursuit. In this the looking procedure will done by positioning procedure. The downside of this idea is a direct result of positioning procedure in house preparing time will be augmented.
The examination on security protecting information mining has multiplicative bother techniques, which are like the Grate encryption, however with more accentuation on safeguarding the utility for information mining.

\section{Methodology}

\section{Inquiry Services in the Cloud}

Inquiry is for the most part used to seek. Inquiries are built by utilizing organized inquiry dialect. It is for the most part used to recovering the required data from the database. Inquiry administrations are the strategy for administrations that are uncovered through an execution of specialist co-op. Here by utilizing RASP, go question and kNN inquiry in cloud give secure, quick putting away and recovering procedure of encryption and unscrambling of an information from database.

Range inquiry is a critical kind of question for some information investigative assignments from basic total to additional complex machine learning assignments. Give $\mathrm{T}$ a chance to be a table and $\mathrm{Xi}, \mathrm{Xj}$, and $\mathrm{Xk}$ be the genuine esteemed qualities in $\mathrm{T}$, and an and $\mathrm{b}$ be a few constants. Take the tallying inquiry for instance. A commonplace range inquiry resembles select tally $(*)$ from $\mathrm{T}$

where $\mathrm{Xi} \in[\mathrm{ai}, \mathrm{bi}]$ and $\mathrm{Xj} \in(\mathrm{aj}, \mathrm{bj})$ and $\mathrm{Xk}=\mathrm{ak}$

which ascertains the quantity of records in the range characterized by conditions on $\mathrm{Xi}, \mathrm{Xj}$, and $\mathrm{Xk}$. Range questions might be connected to self-assertive number of qualities and conditions on these properties joined with restrictive administrators "what's more, "or." We call each piece of the question condition that includes just a single characteristic as a basic condition. A straightforward condition like $\mathrm{Xi} \epsilon$ [ai,bi] can be portrayed with two half space conditions $\mathrm{Xi} \leq$ bi and $-\mathrm{Xi} \leq-$ ai. Without loss of generality, we will talk about how to process half-space conditions like $\mathrm{Xi} \leq$ bi in this paper. A slight alteration will broaden the talked about calculations to deal with different conditions like $\mathrm{Xi}<\mathrm{bi}$ also, $\mathrm{Xi}=$ bi. $\mathrm{kNN}$ inquiry is to locate the nearest $\mathrm{k}$ records to the inquiry point, where the euclide an separation is regularly used to measure the vicinity. It is regularly utilized as a part of location based administrations for looking through the items near a question point, and additionally in machine learning calculations, for example, progressive grouping and $\mathrm{kNN}$ classifier. A kNN question comprises of the inquiry point and the quantity of closest neighbors, $\mathrm{k}$.

\section{Framework Architecture}

We accept that a distributed computing foundation, for example, Amazon EC2, is utilized to have the question administrations and expansive informational indexes. The reason for this design is to expand the exclusive database servers to people in general cloud, or utilize a cross breed private- open cloud to accomplish adaptability and lessen costs while looking after privacy. Each record $\mathrm{x}$ in the outsourced database contains two sections: the RASP-handled characteristics $D^{\prime}=F(D, K)$ and the scrambled unique records, $Z=E\left(D, K^{\prime}\right)$, where $K$ and $K^{\prime}$ are keys for annoyance and encryption, individually. The Grate annoyed information $\mathrm{D}^{\prime}$ are for ordering and inquiry handling. Fig. 1 demonstrates the framework design for both Grate based range inquiry administration and $\mathrm{kNN}$ benefit. 


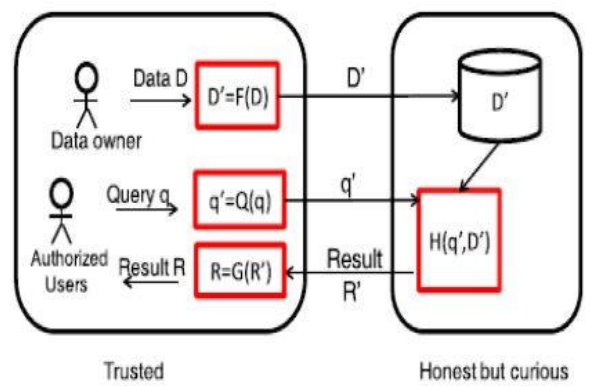

Fig. 1: The system architecture for RASP- based query services

There are two unmistakably isolated gatherings: the trusted parties and the untrusted parties. The trusted gatherings incorporate the information/benefit proprietor, the in-house intermediary server, and the approved clients who can just submit questions. The information proprietors ends out the irritated information to the cloud. Then, the approved clients can submit run questions or kNN inquiries to learn insights or discover a few records. The untrusted parties incorporate the inquisitive cloud supplier who has the inquiry administrations and the secured database. The RASP-irritated information will be utilized to construct records to help question preparing. There are various essential techniques in this structure:

1) F(D) is the RASP irritation that changes the first information D to the annoyed information $\left.D^{\prime} ; 2\right) \mathrm{Q}(\mathrm{q})$ changes the first inquiry q to the secured frame q' that can be prepared on the annoyed information; and 3) $\mathrm{H}\left(\mathrm{q}^{\prime}, \mathrm{D}^{\prime}\right)$ is the question handling calculation that profits the outcome R'. At the point when the measurements, for example, SUM or AVG of a particular measurement are required, RASP can work with fractional homomorphic encryption, for example, Paillier encryption [24] to figure these measurements on the encoded information, which are then recuperated with the technique $G^{\prime}\left(R^{\prime}\right)$.

\section{Danger Model}

The cloud server is considered as "genuine however inquisitive" in our model, which is steady with related takes a shot at cloud security. In particular, the cloud server acts in a "fair" form and accurately takes after the assigned convention determination. Be that as it may, it is "interested" to derive and investigate information (counting file) in its stockpiling and message streams gotten amid the convention to take in extra data.

Suppositions: Our security investigation is based on the critical highlights of the engineering. Under this setting, we trust the accompanying presumptions are proper:

$\square$ Only the approved clients can question the Restrictive database. Approved clients are not pernicious and won't deliberately rupture the classification. We consider insider assaults are orthogonal to our inquire about; in this way, we can bar the circumstance that the approved clients intrigue with the untrusted cloud suppliers to release extra data.

$\square$ The customer side framework and the correspondence channels are legitimately secured and no ensured information records and questions can be spilled.

$\square$ Adversaries can see the irritated database, the changed inquiries, the entire inquiry preparing strategy, the entrance designs, and comprehend the same question restores a similar arrangement of results, yet nothing else.

$\square$ Adversaries can have the worldwide data of the database, for example, the applications of the database, the characteristic spaces, and potentially the characteristic appropriations, by means of other distributed sources (e.g., the circulation of offers, or patient sicknesses, out in the open reports). Secured resources:
Data secrecy and inquiry security ought to be ensured in the RASP approach. While the honesty of question administrations is likewise an imperative issue, it is orthogonal to our investigation. Existing respectability checking and avoiding methods [33], [29], [18] can be incorporated into our structure. Consequently, the uprightness issue will be prohibited from the paper, and we can accept the inquisitive cloud supplier is occupied with the information and inquiries, however it will sincerely take after the convention to give the foundation benefit. Assailant displaying. The objective of assault is to recoup (or assess) the first information from the annoyed information, or recognize the correct inquiries (i.e., area questions) to rupture clients' protection. As indicated by the level of earlier information the assailant may have, we order the assaults into two classes:

$\square$ Level 1: The assailant knows just the irritated

information and changed inquiries, with no other earlier learning. This relates to the cipertext-just assault in the cryptographic setting.

$\square$ Level 2: The assailant additionally knows the first information circulations, including singular quality conveyances and the joint dispersion (e.g., the covariance framework) between characteristics. By and by, for a few applications, whose insights are intriguing to general society space, the dimensional circulations may have been distributed by means of different sources.

\section{Grate: Random Space Perturbation}

Grate indicates Random Space Perturbation. Grate is one sort of multiplicative annoyance, with a novel mix of OPE, measurement extension, arbitrary commotion infusion, and irregular projection. Arbitrary projection is principally used to process the high dimensional information into low dimensional information portrayals. It contains highlights like great scaling potential and great exhibitions. Arbitrary commotion infusion is basically used to adding clamor to the contribution to get appropriate yield when we contrast it with the assessed control. The RASP strategy and its blend give privacy of information and this approach is for the most part used to ensure the multidimensional scope of questions in secure way and furthermore with ordering and productive inquiry preparing will be finished. Grate has some vital highlights. In RASP the utilization of lattice duplication does not secure the dimensional esteems so no compelling reason to experience the ill effects of the dispersion based assault. Grate keeps the information that are irritated from separate based assaults; it doesn't secure the separations that are happened between the records. And furthermore it won't secure more troublesome structures it might be a network and different parts. The range inquiries can be send to the RASP irritated information and this range question portrays open limits in the multidimensional space. In irregular space annoyance, the word irritation is utilized to do falling this procedure will occur as per the key esteem that is given by the proprietor. In this module the information proprietor need to enroll as proprietor and need to give proprietor name and key esteem. And afterward the client have enroll and get the key esteem and information proprietor name from the proprietor to do access in the cloud. Here client can present their inquiry as range question or $\mathrm{kNN}$ question and find their solution. We investigate and demonstrate the outcome with scrambled and furthermore in unscrambled arrangement of the information for the question build by the client. Grate has a few vital highlights. To begin with, RASP does not safeguard the request of dimensional esteems due to the lattice increase segment, which separates itself from arrange protecting encryption plans, and along these lines does not experience the ill effects of the dissemination based assault. Second, RASP does not save the separations between records, which keeps the irritated information from remove based assaults. Since none of the changes in the RASP: Eope, G, and F jelly separations, evidently the RASP irritation won't safeguard 
separations. Third, the first range questions can be changed to the RASP annoyed information space, which is the premise of our inquiry handling methodology. A range inquiry portrays a hypercubic zone (with potentially open limits) in the multidimensional space.

\section{Knn Query Processing with RASP}

The RASP irritation does not save separations (and separation orders), kNN inquiry can't be specifically prepared with the RASP annoyed information. In this area, we plan a kNN inquiry handling calculation in view of range questions (the kNN-R calculation). Subsequently, the utilization of record in extend question handling likewise empowers quick preparing of $\mathrm{kNN}$ inquiries. The first separation based kNN inquiry preparing finds the closest $\mathrm{k}$ focuses in the circular range that is focused at the question point. The essential thought of our calculation is to utilize square ranges, rather than circular reaches, to locate the rough $\mathrm{kNN}$ comes about, with the goal that the RASP run question administration can be utilized. There are various key issues to make this work safely and proficiently. 1) How to proficiently locate the base square range that most likely contains the $\mathrm{k}$ comes about, without numerous connections between the cloud and the customer? 2) Will this arrangement safeguard information secrecy and inquiry protection? 3) Will the intermediary server's workload increment? what exactly degree? The calculation depends on square ranges to roughly discover the kNN contender for a question point, which are characterized as takes after.

Definition 1: "A square range is a hypercube that is focused at the question point and with rise to length edges."

Fig. 2 delineates the range-inquiry based kNN handling with 2D information. The Inner Range is the square range that contains in any event $\mathrm{k}$ focuses, and the Outer Range encases the round range that encases the inward range. The external run doubtlessly contains the kNN comes about (see Proposition 2) yet it might likewise contain superfluous focuses that should be separated out. Suggestion 1 : "The kNN-R calculation returns comes about with 100 percent review."

Verification: The circle in Fig. 2 between the external range and the inward range covers all focuses with separations not exactly the span $r$. Since the internal range contains in any event $k$ focuses, there are in any event $\mathrm{k}$ closest neighbors to the inquiry focuses with removes not as much as the range $\mathrm{r}$. Thusly, the $\mathrm{k}$ closest neighbors must be in the external range.

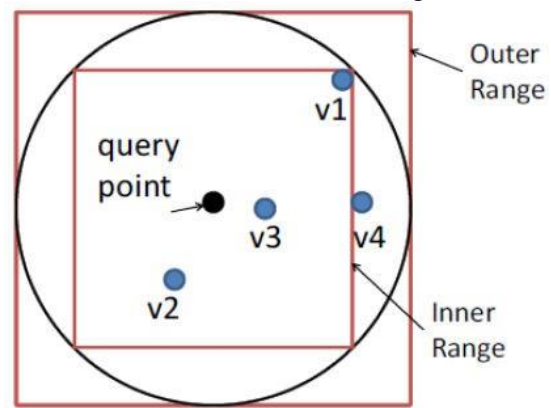

Fig. 2: Illustration for kNN-R Algorithm when $\mathrm{k}=3$.

The kNN-R calculation comprises of two rounds of connections between the customer and the server. Fig. 3 exhibits the strategy. 1) The customer will send the underlying upper bound range, which contains more than $\mathrm{k}$ focuses, and the underlying lower bound range, which contains not as much as $\mathrm{k}$ focuses, to the server. The server finds the inward range and comes back to the customer. 2) The customer ascertains the external range in light of the inward range and sends it back to the server. The server finds the records in the external range and sends them to the customer. 3) The customer unscrambles the records and locate the best $\mathrm{k}$ hopefuls as the last outcome.[13]

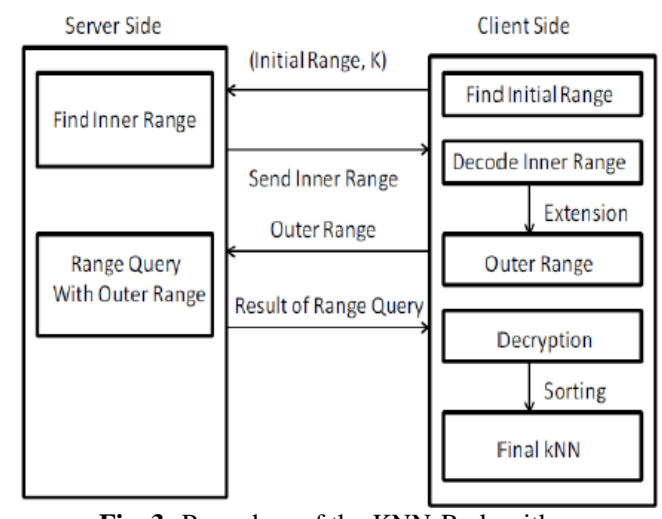

Fig. 3: Procedure of the KNN-R algorithm

In the event that the focuses are around consistently circulated, we can appraise the exactness of the returned result. With the uniform supposition, the quantity of focuses in a region is corresponding to the measure of the zone. On the off chance that the internal go contains $m$ focuses, $m>=k$, the external range contains $q$ focuses, and the dimensionality is $\mathrm{d}$, we can infer $\mathrm{q}=2 \mathrm{~d}=2 \mathrm{~m}$. [14]

\section{Conclusion}

We propose to think about an outsourced benefit in view of the CPEL criteria: information Confidentiality, question Privacy, Efficient question preparing, and Low in house workload. With the CPEL criteria as a main priority, we build up the kNN-R approach for secure outsourced kNN question benefit. The kNN-R approach exploits quick and secure RASP extend question preparing to execute $\mathrm{kNN}$ question handling. It can discover high accuracy kNN comes about and furthermore limit the connections between the cloud server and the in house customer. High accuracy kNN comes about and limited collaborations result in low in house workload. We have led a careful security examination on information secrecy and inquiry protection. Contrasted with the related methodologies, the kNN-R approach accomplishes a superior adjust over the CPEL criteria. Scratch technique with extend inquiry and kNN question. This strategy essentially used to annoy the information given by the proprietor what's more, spared in distributed storage it additionally joins arbitrary infusion, arrange safeguarding encryption and arbitrary commotion projection and additionally it has contains CPEL criteria in it. By utilizing the range inquiry and kNN question client can recover their information's in secured way and the process in time of the question is limited.

\section{References}

[1] Xu H, Guo S \& Chen K, Building confidential and efficient query services in the cloud with RASP data perturbation", IEEE Transactions on Knowledge and Data Engineering, Vol.26, No.2, (2014).

[2] Chen K, Kavuluru R \& Guo S, "RASP: Efficient Multidimensional Range Query on Attack-Resilient Encrypted Databases", Proc. ACM Conf. Data and Application Security and Privacy, (2011), pp.249-260.

[3] Agrawal R, Kiernan J, Srikant R \& Xu Y, "Order Preserving Encryption for Numeric Data", Int'l Conf. Management of Data (SIGMOD), (2004).

[4] Armbrust M, Fox A, Griffith R, Joseph AD, Andy Konwinski RK, Lee G, Patterson D, Rabkin A, Stoica I \& Zaharia M, "Above the Clouds: A Berkeley View of Cloud Computing”, Technical report, Univ. of Berkerley, (2009).

[5] Bau J \& Mitchell JC, "Security Modeling and Analysis", IEEE Security and Privacy, Vol.9, No.3, (2011), pp.18-25.

[6] Cao N, Wang C, Li M, Ren K \& Lou W, "Privacy-Preserving Multi-Keyword Ranked Search over Encrypted Cloud Data", Proc. IEEE INFOCOMM, (2011).

[7] Chen K \& Liu L, "Geometric Data Perturbation for Outsourced 
Data Mining", Knowledge and Information Systems, Vol.29, (2011), pp.657- 695.

[8] G, Abikhanova, A Ahmetbekova, E Bayat, A Donbaeva, G Burkitbay (2018). International motifs and plots in the Kazakh epics in China (on the materials of the Kazakh epics in China), Opción, Año 33, No. 85. 20-43.

[9] D, Ibrayeva, Z Salkhanova, B Joldasbekova, Zh Bayanbayeva (2018). The specifics of the art autobiography genre. Opción, Año 33. 126-151.

[10] Chen K, Liu L \& Sun G, "Towards Attack-Resilient Geometric Data Perturbation", Proc. SIAM Int'l Conf. Data Mining, (2007).

[11] Chor EK, Goldreich O \& Sudan M, "Private Information Retrieval”, ACM Computer Survey, Vol.45, No.6, (1998), pp.965981.

[12] Curtmola R, Garay J, Kamara S \& Ostrovsky R, "Searchable Symmetric Encryption: Improved Definitions and Efficient Constructions", 13th ACM Conf. Computer and Comm. Security, (2006), pp.79- 88

[13] Marimont R \& Shapiro M, "Nearest Neighbour Searches and the Curse of Dimensionality", J. Inst. of Math. and Its Applications, Vol.24, (1979), pp.59-70

[14] Hacigumus H, Iyer B, Li C \& Mehrotra S, "Executing SQL over Encrypted Data in the Database-Service-Provider Model", $A C M$ SIGMOD Int'l Conf. Management of Data (SIGMOD), (2002). 